\title{
Impacto do alongamento muscular no desempenho motor de indivíduos ativos
}

\author{
Impact of muscle stretching in active individual engine performance \\ Impacto del estiramiento muscular en el rendimiento motor de personas activas
}

Francisco Robson de Oliveira Alves

ORCID: https://orcid.org/0000-0002-9854-6460

Universidade Federal do Piauí, Brasil

E-mail: robson_oliveira.fisio@outlook.com

Ana Mara Ferreira Lima

ORCID: https://orcid.org/0000-0001-9039-7328

Christus Faculdade do Piauí, Brasil

E-mail: anamarafl@yahoo.com.br

Telmo Macedo de Andrade

ORCID: https://orcid.org/0000-0003-1313-2259 Universidade Maurício de Nassau, Brasil

E-mail: telmo_andrade@ hotmail.com

Cibelle Maria Sampaio Alves de Andrade

ORCID: https://orcid.org/0000-0001-6588-401X

Hospital de Urgências de Teresina, Brasil

E-mail: cibelle_sampaio@hotmail.com

\begin{abstract}
Resumo
$\mathrm{O}$ alongamento muscular é frequentemente realizado antes ou após às práticas de atividade física, com o intuito de aumentar a amplitude articular e a flexibilidade muscular. Alguns estudos recentemente publicados afirmam que o alongamento pré-exercício compromete a produção de força muscular e interfere no desempenho do exercício. Buscou-se avaliar o impacto do alongamento na flexibilidade e força muscular de indivíduos praticantes de AF. Tratou-se de uma pesquisa do tipo descritiva, com abordagem quantitativa exploratória. Os testes e intervenções foram realizados em apenas um grupo, com a presença de um grupo controle, e com uma amostra não-aleatória selecionada por voluntariedade. A amostra foi composta por 40 indivíduos. Todos foram avaliados no quesito desempenho motor nas variáveis força muscular de flexão de cotovelo pela dinamometria; flexibilidade pelo TSA; e na performance física com o teste de salto horizontal. Posteriormente, o grupo experimental passou por intervenções semanais de alongamento, bem como, receberam orientações para uma mudança na rotina de AF, bem como para incluírem de forma integral a técnica de alongamento. Ao realizar a comparação, dos valores das variáveis ao final da pesquisa, entre os sexos masculino e feminino percebe-se que há diferença entre a força muscular de MSD e MSE, flexibilidade e desempenho físico entre homens e mulheres tanto no GC ( $\mathrm{p}=0,001801613$; $\mathrm{p}=0,658869802$; $\mathrm{p}=0,184851508 ; \mathrm{p}=0,000$ respectivamente $)$ quanto no GI $(\mathrm{p}=0,008975882 ; \mathrm{p}=0,071945664 ; \mathrm{p}=0,039723589$; $\mathrm{p}=0,0056328$ respectivamente) e que estes valores apresentaram-se estatisticamente significativos. A técnica pode potencializar o treinamento, permitindo que seu praticante tenha um maior aproveitamento e obtenha maiores resultados.
\end{abstract}

Palavras-chave: Exercícios de alongamento muscular; Fisioterapia; Atividade motora; Desempenho psicomotor.

\begin{abstract}
Muscle stretching is often performed before or after physical activity practices in order to increase joint range and muscle flexibility. Some recently published studies state that pre-exercise stretching compromises muscle strength production and interferes with exercise performance. This study aimed to evaluate the impact of stretching on flexibility and muscle strength of individuals who practice PA. This was a descriptive research with a quantitative exploratory approach. The tests and interventions were performed in only one group, with the presence of a control group, and with a non-random sample selected voluntarily. The sample was composed of 40 individuals. All were evaluated in terms of motor performance in the variables: muscle strength in elbow flexion by dynamometry; flexibility by TSA; and physical performance with the horizontal jump test. Afterwards, the experimental group underwent weekly stretching interventions, and received orientations for a change in the PA routine, as well as to include the stretching technique in an integral way. When comparing the values of the variables at the end of the study, between the male and female sexes, it was noticed that there was a difference between the MSD and MSE muscle strength, flexibility, and physical performance between men and women in both the $C G(p=0.001801613 ; p=$
\end{abstract}


$0.658869802 ; \mathrm{p}=0.184851508 ; \mathrm{p}=0.000$ respectively $)$ and in $\mathrm{GI}(\mathrm{p}=0.008975882 ; \mathrm{p}=0.071945664 ; \mathrm{p}=0.039723589$; $\mathrm{p}=0.0056328$ respectively) and that these values were statistically significant. The technique can potentiate the training, allowing its practitioner to have a greater utilization and obtain greater results.

Keywords: Muscle stretching exercises; Physiotherapy; Motor activity; Psychomotor performance.

\section{Resumen}

Los estiramientos musculares suelen realizarse antes o después de las prácticas de actividad física para aumentar la amplitud articular y la flexibilidad muscular. Algunos estudios publicados recientemente afirman que los estiramientos previos al ejercicio comprometen la producción de fuerza muscular e interfieren en el rendimiento del ejercicio. Este estudio pretendía evaluar el impacto de los estiramientos en la flexibilidad y la fuerza muscular de los individuos que practican AF. Se trata de una investigación descriptiva con un enfoque exploratorio cuantitativo. Las pruebas e intervenciones se realizaron en un solo grupo, con la presencia de un grupo de control, y con una muestra no aleatoria seleccionada voluntariamente. La muestra estaba compuesta por 40 individuos. Todos fueron evaluados en términos de rendimiento motor en las variables fuerza muscular de flexión del codo por dinamometría; flexibilidad por TSA; y rendimiento físico con la prueba de salto horizontal. Posteriormente, el grupo experimental se sometió a intervenciones semanales de estiramiento y recibió orientación para un cambio en la rutina de AF, así como para incluir completamente la técnica de estiramiento. Al realizar la comparación, de los valores de las variables al final de la investigación, entre los sexos masculino y femenino se observa que hay diferencia entre la fuerza muscular de MSD y MSE, la flexibilidad y el rendimiento físico entre hombres y mujeres tanto en GC ( $\mathrm{p}=0,001801613 ; \mathrm{p}=$ 0,$658869802 ; \mathrm{p}=0,184851508 ; \mathrm{p}=0,000$ respectivamente) como en GI ( $\mathrm{p}=0,008975882 ; \mathrm{p}=0,071945664$; $\mathrm{p}=0,039723589 ; \mathrm{p}=0,0056328$ respectivamente) y que estos valores presentaron significación estadística. La técnica puede potenciar el entrenamiento, permitiendo que su practicante tenga un mayor aprovechamiento y obtenga mayores resultados.

Palabras clave: Ejercicios de estiramiento muscular; Fisioterapia; Actividad motriz; Rendimiento psicomotor.

\section{Introdução}

O alongamento é um termo geral usado para descrever qualquer técnica fisioterapêutica elaborada com o objetivo de aumentar a extensibilidade dos tecidos moles, melhorando a flexibilidade com o aumento do tamanho das estruturas que, de modo adaptativo, encurtaram-se e tornaram-se hipomóveis com o tempo (Kisner \& Colby, 2009).

Existem diversas técnicas de alongamento entre as quais pode-se citar o alongamento balístico, estático e contrairrelaxar. O balístico é caracterizado pela movimentação rítmica e vigorosa de um segmento do corpo para promover o alongamento da musculatura. O estático caracteriza-se pelo alongamento dos tecidos moles até o ponto de resistência na mesma posição, enquanto a técnica do contrair-relaxar se dá por uma leve contração isométrica do músculo agonista, que inibe o músculo para o alongamento, e quando relaxado, o mesmo é alongado (Morcelli, Oliveira \& Navega, 2013).

Atividade física denomina-se como qualquer movimento corporal que os músculos esqueléticos produzem, resultando em gasto energético maior que níveis de repouso. Ela abrange duas vertentes que enfatizam os motivos para a prática, a primeira contempla características biológicas (capacidade aeróbica, controle ponderal, flexibilidade, força e resistência muscular) que diminuem as consequências do sedentarismo se comparados com indivíduos ativos; e a segunda está relacionada com desempenho, envolve uma série de componentes ligados a atividades laborais, como o equilíbrio, coordenação e reação muscular (Maciel, 2010).

A força e a flexibilidade são componentes importantes dos protocolos de treinamento físico podendo impactar na qualidade de vida, capacidade funcional na saúde e no desempenho motor. Costuma-se usar sessões complexas e é comum combinar exercícios de força e flexibilidade na prescrição do treinamento físico-esportivo. Dessa forma, é fundamental que se compreenda a influência de uma capacidade motora sobre a outra, sendo relevante para a correta prescrição de exercícios, possibilitando a diminuição dos efeitos deletérios que possam ocorrer sobre o sistema muscular (Paulo et al., 2012).

Adaptações musculoesqueléticas relacionadas ao exercício de alongamento dependem da frequência, duração e intensidade com que o alongamento é efetuado. Isso promove o desenvolvimento de respostas plásticas e elásticas dessas estruturas, interferindo diretamente nas variáveis flexibilidade e geração de força muscular total (Salvini et al., 2012). 
Quando se quer avaliar níveis de flexibilidade, o teste de sentar e alcançar (TSA) é bastante empregado, pois é um indicador confiável da flexibilidade dos músculos ísquiosurais e da coluna lombar. Vícios posturais e dores na coluna vertebral estão relacionados com o baixo desempenho em exames de aptidão física, que refletem em não atender os critérios de saúde adotados por diferentes baterias de testes (Coledam, Arruda \& Oliveira, 2013).

Devido às diversas formas de manifestação, a força é analisada por diferentes meios de avaliação, porém, a forma mais objetiva e simples se faz necessária para que se obtenha informações concisas para investigações clínico-científicas. Como ferramentas de avaliação tem-se quatro grandes grupos de testes para mensurar força: as escalas de graduação manual de força, como a Escala de Oxford, os testes de impulso ou salto (horizontal, vertical ou sêxtuplo), a dinamometria, seja com o uso de dinamômetros isocinéticos ou portáteis, e os testes de repetição máxima (Camargo et al.,2009).

Os saltos horizontal, vertical e sêxtuplo têm sido utilizados como indicadores da força dos membros inferiores (MMII), uma vez que demonstraram sensibilidade para diferentes tipos de treinamento de força. Esses testes de impulsos são utilizados por serem específicos às ações de diferentes modalidades esportivas, sendo que os tipos vertical e sêxtuplo são indicadores mais específicos para velocidade e agilidade, ficando com o horizontal, a maior representação para o aspecto força muscular (Oliveira et al., 2013).

A dinamometria portátil é um procedimento seguro, rápido, é um instrumento acessível à maioria dos clínicos e não exige treinamento extensivo. Essa medida de preensão palmar tem relação direta com a recuperação de membro superior, como observado no movimento de alcance e da perspectiva da força muscular geral do corpo (Soares et al., 2011).

Alguns estudos recentemente publicados afirmam que o alongamento pré-exercício compromete a produção de força muscular e interfere no desempenho do exercício. Em contrapartida, outros constatam que os indivíduos que se alongam regularmente apresentam melhor velocidade de contração das fibras musculares, proporcionando maior força na contração concêntrica e excêntrica (Lustosa et al., 2010). Partindo desse pressuposto, busca-se avaliar o impacto do alongamento na flexibilidade e força muscular de indivíduos praticantes de AF.

Durante décadas, sabe-se da importância do alongamento antes e após a prática de qualquer AF e tem sido um método comum em todos os níveis de exercícios. Contudo, atualmente há muita discussão e se tem demonstrado que o alongamento antes do exercício não diminui os índices de lesões e que interfere no rendimento do desempenho físico dos indivíduos que o praticam.

Segundo os novos dados, em alguns indivíduos, tal prática poderia predispor a lesão, ou seja, é provável que haja pouca ou nenhuma vantagem na prevenção e diminuição de risco de lesões quando o atleta, amador ou profissional, realiza alongamento antes do treinamento. Dessa forma, faz-se necessários estudos que possam verificar na prática qual a melhor decisão deve ser tomada ao se preparar para o exercício físico, se o indivíduo deve ou não se alongar.

\section{Metodologia}

Tratou-se de uma pesquisa do tipo descritiva, comparativa de caráter prospectivo, com abordagem quantitativa exploratória. Os testes e intervenções foram realizados em apenas um grupo, com a presença de um grupo controle, e com uma amostra não-aleatória selecionada por voluntariedade. Um grupo recebeu intervenção e o outro não, depois foram analisados, comparados e correlacionados.

A amostra foi composta por 40 indivíduos. A seleção se deu por método não-probabilístico, voluntário, por meio de comunicação oral a partir do público de estudantes da Christus Faculdade do Piauí (CHRISFAPI). Os indivíduos foram informados sobre os procedimentos metodológicos desta investigação e, aqueles que aceitaram participar assinaram um Termo de Consentimento Livre e Esclarecido (TCLE), em que foi manifestado o conhecimento da proposta metodológica do projeto e 
o interesse em fazer parte da amostra. Os voluntários compareceram à Fundação Escola Chrisfapi em datas e horários préestabelecidos para a coleta de dados e aplicação das intervenções. Foram incluídos na pesquisa indivíduos com idade entre $18 \mathrm{e}$ 60 anos; que concordarem em participar da pesquisa mediante assinatura do TCLE; que já praticam AF há mais de 2 anos e que essa prática seja de pelo menos 3 vezes por semana. Sendo excluídos, poranto, indivíduos com idade inferior a 18 e superior a 60 anos; sedentários; que praticam AF há menos de 2 vezes por semana; e os que se recusarem a assinar o TCLE, por qualquer motivo.

Para mensurar a força muscular de flexão de cotovelo, utilizou-se um dinamômetro, o FORCE GAUGE DD-500 INSTRUTHERM (5000g x 1g; 49,03 × 0,01 N; 176,40 × 0,05 Ib). Para mensurar a distância alcançada no TSA usou-se uma caixa de madeira com uma escala de 26,0 centímetros $(\mathrm{cm})$ em seu prolongamento com dimensões de $30,5 \mathrm{~cm}$ x $30,5 \mathrm{~cm} \times$ $30,5 \mathrm{~cm}$. E para mensurar a distância do salto horizontal utilizou-se uma fita métrica.

As coletas de dados foram realizadas na sala do ginásio da Clínica Escola da CHRISFAPI, mediante a assinatura da Declaração de Autorização da Instituição Coparticipante, no turno vespertino no intervalo de horário das 13 às 18 horas, com agendamento dos participantes, para não desperdiçar o tempo dos mesmos. Os sujeitos da amostra estavam cientes de que deveriam comparecer a todos os encontros marcados com roupas leves e confortáveis e sem atrasos.

Os participantes se apresentaram na Clínica Escola da Chrisfapi no horário determinado pelo pesquisador. Foram conduzidos ao consultório e lá tiveram sua massa corporal e estatura medidas, passaram por uma anamnese em ficha própria e foram anotadas todas as informações necessárias. Nesse dia também receberam orientações de técnicas de autoalongamento e foram repassados os procedimentos metodológicos da pesquisa. Nesse mesmo dia, os indivíduos assinaram o TCLE.

O protocolo de exercício utilizado foi alongamento global e específico para a musculatura flexora, extensora, abdutora e adutora de membros superiores e inferiores, bem como da flexora e extensora da toraco-lombar 2 (duas) vezes por semana, por um período de 30 (trinta) dias.

Protocolo segundo Kisner e Colby (2009):

- Alongamento estático: Uma força externa no final da AM, mantida, aplicada com pressão adicional e por meio de contato manual, 2 (duas) repetições de 30 (trinta) segundos de duração cada;

- Técnica de contrair e relaxar: o músculo que limita AM é primariamente alongado até o ponto da limitação ou até o ponto mais confortável para o paciente, que faz, então, uma contração isométrica de 10 (dez) segundos no final da amplitude, antes do alongamento 10 (dez) segundos, seguida pelo relaxamento do músculo encurtado.

- Autoalongamento: Qualquer exercício de alongamento feito independentemente por um paciente após instrução e supervisão de um fisioterapeuta é chamado de autoalongamento. O paciente deve manter cada postura de alongamento orientada pelo pesquisador por um tempo de 30 (trinta) segundos.

Todos foram avaliados no quesito desempenho motor nas variáveis força muscular de flexão de cotovelo pela dinamometria; flexibilidade pelo TSA; e na performance física com o teste de salto horizontal e com os dados anotados em uma ficha própria. Posteriormente, o grupo experimental passou por intervenções semanais de alongamento, bem como, receberam orientações para uma mudança na rotina de $\mathrm{AF}$, bem como para incluírem de forma integral a técnica de alongamento.

O teste utilizado para avaliar a flexibilidade foi o de Sentar e Alcançar proposto originalmente por Dillon e Wells em 1952, seguindo a padronização canadense para os testes de avaliação da aptidão física do Canadian Standardized Test of Fitness (CSTF). O teste é realizado numa caixa medindo $30,5 \mathrm{~cm}$ x $30,5 \mathrm{~cm}$ x 30,5 cm com uma escala de $26,0 \mathrm{~cm}$ em seu prolongamento, sendo que o ponto zero se encontra na extremidade mais próxima do avaliado e o $26^{\circ} \mathrm{cm}$ coincide com o ponto de apoio dos pés. $\mathrm{O}$ avaliado retira o calçado e na posição sentada toca os pés na caixa com os joelhos estendidos. Com ombros flexionados, cotovelos estendidos e mãos sobrepostas deve executar a flexão do tronco à frente devendo este tocar o ponto 
máximo da escala com as mãos. Serão realizadas três tentativas sendo considerada apenas a melhor marca (Ribeiro et al., 2010).

Já para mensurar a força muscular de flexão de cotovelo utilizou-se um dinamômetro, FORCE GAUGE DD-500 INSTRUTHERM $(5000 \mathrm{~g} \times 1 \mathrm{~g} ; 49,03 \times 0,01 \mathrm{~N} ; 176,40 \times 0,05 \mathrm{Ib})$. No momento dos testes, o paciente estará posicionado sentado confortavelmente, com o ombro aduzido, cotovelo fletido a $90^{\circ}$, antebraço e punho em posição neutra. Quatro mensurações são realizadas em cada membro, sendo a primeira utilizada para adaptação e conhecimento do equipamento e, por conta disso, descartada. Com as demais medidas calcula-se a média aritmética (Soares et al., 2011).

Para avaliar o desempenho físico no salto horizontal o indivíduo fica em pé, com pés ligeiramente afastados e paralelos, ponta dos pés logo atrás da linha que será delimitada no chão. Em seguida o indivíduo realiza um movimento de balanceio dos braços - à vontade - como movimento preparatório, semiflexionando os joelhos. O salto é realizado lançando os braços para frente, estendendo o quadril, joelhos e tornozelos - por consequência do balanceio permitido, o indivíduo se utiliza da energia elástica acumulada após a rápida extensão mecânica dos músculos extensores seguida do movimento descrito acima - O objetivo é alcançar a máxima projeção horizontal. O procedimento deve ser realizado uma única vez (Falk \& Pereira, 2009).

A coleta de dados foi do tipo fracionada, a primeira, aconteceu nos meses de novembro e dezembro de 2017 (22 indivíduos analisados) e a segunda, entre março e abril de 2018 (18 indivíduos analisados). Foi iniciada por meio de uma entrevista realizada individualmente e em ambiente confidencial, realizada pelo próprio pesquisador para fins de preenchimento de uma ficha de avaliação, impressa e elaborada pelo próprio autor, contemplando características e dados sócio demográficos como: identificação, dados antropométricos (altura, peso) e hábitos de vida (tabagista, etilista, tipo de AF que realiza, hipertensão, alterações musculoesqueléticas e cirurgias anteriores), e tinha os espaços das variáveis avaliadas para anotação e produção de dados. Os dados produzidos e obtidos foram tabulados em tabelas e colocados em planilhas no programa Microsoft Office Excel 2013.

Os dados tabulados em tabelas foram analisados por bases estatísticas descritivas simples no programa Microsoft Office Excel 2013 e Minitab 18. Avaliou-se a consistência e significância estatística e verificou-se a normalidade dos dados com o teste de Kolmogorov-Smirnov e logo em seguida foi usado o Teste $t$ de Student.

Para verificar se houve diferenças significativas das medidas das variáveis avaliadas antes e depois do período relativo a pesquisa e entre os grupo controle (GC) e grupo intervenção (GI), foi utilizado o cálculo amostral do programa Minitab 18. Para os testes utilizou-se um poder de confiabilidade de $95 \%$. Adotou-se um nível de significância de $5 \%$ ( $\mathrm{p} \leq 0.05)$.

A coleta de dados só foi iniciada após a aprovação pelo Comitê de Ética em Pesquisa envolvendo Seres Humanos (CEP) da Associação Teresinense de Ensino - Faculdades Santo Agostinho/ATE sob o número CAAE 72311817.0.0000.5602 e número do parecer 2.294.313, obedecendo aos preceitos norteadores da resolução 466/12 do Conselho Nacional de Saúde (CNS), ressaltando-se que em relação a eticidade da pesquisa, que a mesma respeitou o participante em sua dignidade e autonomia, reconhecendo sua vulnerabilidade, assegurando sua vontade de contribuir e permanecer, ou não, na pesquisa, por intermédio de manifestação expressa livre e esclarecida.

Para garantir o sigilo dos participantes, todos os dados foram utilizados para fins exclusivos da pesquisa, não sendo mencionados os nomes dos pesquisados e assegura-se que os mesmos foram isentos de quaisquer despesas durante a sua colaboração com o presente estudo.

Os dados coletados ficaram sob responsabilidade do pesquisador que manterá esses dados em arquivo físico ou digital, sob sua guarda por um período de 5 anos após o término da pesquisa no acervo da biblioteca da Christus Faculdade do Piauí (CHRISFAPI). 


\section{Resultados e Discussão}

Para a realização deste estudo foram selecionados 40 participantes (20 homens e 20 mulheres). O público foi de estudantes da Christus Faculdade do Piauí (CHRISFAPI), localizada no município de Piripiri-PI. A coleta foi fracionada; a primeira, aconteceu nos meses de novembro e dezembro de 2017 e a segunda, entre março e abril de 2018.

Antes de realizar a mensuração das variáveis analisadas (força muscular, flexibilidade e desempenho físico), foi preenchida uma ficha de avaliação que continha a identificação do paciente com dados sociodemográficos (nome, idade, sexo e profissão), valores antropométricos (altura e peso) e antecedentes mórbidos (processo álgico ou degenerativo, traumatismo musculoesquelético, desequilíbrio biomecânico ou anatômico).

Os participantes foram divididos em dois grupos de 20 indivíduos, um GC com 10 homens e 10 mulheres e um GI, com as mesmas características e a mesma amostra do GC, sendo 10 homens e 10 mulheres. Todos foram avaliados inicialmente e reavaliados após quatro semanas, sendo que somente o GI recebeu os alongamentos, enquanto o GC, privou-se de qualquer técnica relacionada durante o período da coleta.

Quanto as características sociodemográficas no que diz respeito a profissão, 82,5\% eram estudantes, 5\% enfermeiros, 2,5\% fisioterapeutas e 10\% entre outras profissões (auxiliar administrativo, auxiliar de enfermagem, auxiliar de serviços gerais e técnico de laboratório químico). Em hábitos e vícios, $45 \%$ dos indivíduos eram fumantes e apenas 5\% consumiam algum tipo de bebida alcoólica. Quanto a prática de AF, 67,5\% do público praticava musculação, 5\% Pilates, 5\% cross fit e 42,5\% praticavam outros esportes (corrida, natação, futebol, capoeira, Muay Tai e Jiu-Jitsu). No aspecto relacionado aos antecedentes mórbidos, 22,5\% eram cirurgiados, prevalecendo 7,5\% de hérnia umbilical, 5\% de apendicectomia, 2,5\% de cesáreas e 7,5\% de outras cirurgias (redução óssea pós-fratura e colecistectomia). Em relação aos traumas musculoesqueléticos, 10\% possuíam fraturas, $10 \%$ instabilidade articular, $10 \%$ luxação e 7,5\% algum tipo de ruptura muscular. De todos os participantes, 37,5\% queixavam-se de algia em coluna lombar, $5 \%$ em ombro e $12,5 \%$ em joelho.

Não se encontraram na literatura, pesquisas com metodologia semelhante a usada neste estudo, ou seja, um programa com técnicas de alongamento, com indivíduos ativos, por um período de 4 semanas ( 2 intervenções por semana), avaliados em força muscular, flexibilidade e desempenho físico, para a comparação dos dados encontrados. No entanto, existem vários autores que concordam e discordam da utilização do alongamento para a prática de AF e outros que investigaram os efeitos agudos e crônicos da técnica.

Realizou-se a mensuração da dinamometria de flexores de cotovelo em membro superior direito (MSD) e em membro superior esquerdo (MSE). Foi observado que não houve ganho de força muscular nos GC masculino e controle feminino. Houveram perdas e alguns conseguiram manter um percentual de força dentro do valor encontrado na primeira avaliação. Porém, essa perda não teve significância estatística em nenhum dos membros superiores (MMSS), conforme demonstrado abaixo na Tabela 1 ( $\mathrm{p}=0,9962$ para MSD e $\mathrm{p}=0,328083419$ para MSE) e na Tabela $1(\mathrm{p}=0,881607$ para MSD e $\mathrm{p}=0,473215$ para MSE). 
Tabela 1 - Medidas de dinamometria do GC masculino e feminino antes e depois* do período de intervenção. Piripiri-PI. $(n=20)$.

\begin{tabular}{|c|c|c|c|c|c|}
\hline \multicolumn{6}{|c|}{ Grupo Masculino } \\
\hline \multicolumn{2}{|c|}{$\operatorname{MSD}(\mathbf{k g})$} & \multicolumn{4}{|c|}{ MSE(kg) } \\
\hline ANTES & DEPOIS & p-valor & ANTES & DEPOIS & $\mathrm{p}$-valor \\
\hline MÉDIA $\pm D P$ & MÉDIA $\pm D P$ & & MÉDIA $\pm D P$ & MÉDIA $\pm D P$ & \\
\hline $4910,41 \pm$ & $4909,84 \pm$ & $0,9962 * *$ & $4968,98 \pm$ & $4823,78 \pm$ & $0,328083419^{* *}$ \\
\hline 250,89 & 279,95 & & 297,78 & 437,95 & \\
\hline \multicolumn{6}{|c|}{ GRUPO FEMININO } \\
\hline ANTES & DEPOIS & $\mathrm{p}$-valor & ANTES & DEPOIS & $\mathrm{p}$-valor \\
\hline MÉDIA $\pm D P$ & MÉDIA $\pm \mathrm{DP}$ & & MÉDIA $\pm \mathrm{DP}$ & MÉDIA $\pm D P$ & \\
\hline $4499,04 \pm$ & $4440,09 \pm$ & $0,881607 * *$ & $4599,21 \pm$ & $4523,48 \pm$ & $0,473215^{* *}$ \\
\hline 348,39 & 294,26 & & 408,95 & 204,60 & \\
\hline
\end{tabular}

*Dados apresentados como média, desvio padrão e p-valor.

**Sem diferença significante antes e depois sem aplicação do alongamento.

Fonte: Dados da pesquisa (2018).

Por outro lado, para os valores de dinamometria do GI (masculino e feminino), mostram que houve um aumento considerável de força muscular em ambos os MMSS após a intervenção com as técnicas de alongamento. Pode-se observar que os valores têm significância estatística na Tabela 2 ( $\mathrm{p}=0,02737641$ para MSD e p=0,000797966 para MSE) e Tabela 2 $\mathrm{p}=0,007471$ para MSD e $\mathrm{p}=0,010043855$ para MSE).

Tabela 2 - Medidas de dinamometria do GI masculino e feminino antes e depois* do período de intervenção. Piripiri-PI. $(n=20)$.

\section{Grupo Masculino}

\begin{tabular}{|c|c|c|c|c|c|}
\hline \multicolumn{2}{|c|}{ MSD(kg) } & \multicolumn{4}{|c|}{ MSE(kg) } \\
\hline ANTES & DEPOIS & p-valor & ANTES & DEPOIS & p-valor \\
\hline MÉDIA $\pm D P$ & MÉDIA $\pm \mathrm{DP}$ & & MÉDIA $\pm \mathrm{DP}$ & MÉDIA $\pm \mathrm{DP}$ & \\
\hline $5157,14 \pm$ & $5463,02 \pm$ & $0,02737641 * *$ & $4957,83 \pm$ & $5365,21 \pm$ & $0,000797966^{* *}$ \\
\hline 299,08 & 269,93 & & 239,73 & 212,30 & \\
\hline \multicolumn{6}{|c|}{ GRUPO FEMININO } \\
\hline ANTES & DEPOIS & $\mathrm{p}$-valor & ANTES & DEPOIS & p-valor \\
\hline MÉDIA $\pm D P$ & MÉDIA $\pm \mathrm{DP}$ & & MÉDIA $\pm \mathrm{DP}$ & MÉDIA $\pm \mathrm{DP}$ & \\
\hline $4198,56 \pm$ & $4965,75 \pm$ & $0,007471 * *$ & $4525,27 \pm$ & $5137,64 \pm$ & $0,010043855 * *$ \\
\hline 456,07 & 447,99 & & 488,02 & 307,26 & \\
\hline
\end{tabular}

*Dados apresentados como média, desvio padrão e p-valor.

**Com diferença significante antes e depois com aplicação do alongamento.

Fonte: Dados da pesquisa (2018).

Lustosa et al. (2010) encontraram resultados diferentes em suas pesquisas. Observaram diminuição de força muscular e sugeriram duas hipóteses para explicar essa redução induzida pelo alongamento. A primeira refere-se às propriedades viscoelásticas e fatores mecânicos do músculo. Nesse caso, a diminuição de força provocada pelo alongamento poderia estar relacionada com o aumento da complacência muscular, que alteraria a relação comprimento-tensão o que, por sua vez, diminuiria a produção de força devido à relação força-velocidade. E ainda relacionam-se a uma menor rigidez da unidade 
musculotendínea, sugerindo uma interferência na habilidade em recrutar unidades motoras. A segunda hipótese, refere-se aos fatores neurais que alterariam as estratégias de controle motor ou a resposta reflexa.

Apesar de esses preceitos explicarem a modificação da força muscular, essas evidências fornecem apenas suporte indireto para a hipótese de que o alongamento provoca alterações agudas na geração de força muscular. Por isso essa condição não foi observada neste estudo, pois avaliou-se a interferência do alongamento acumulado nesta variável. Sugerindo assim que existem respostas diferenciadas quando avaliados o efeito agudo do alongamento e o efeito acumulado da intervenção.

Em contrapartida, Shrier (2014), após uma revisão sistemática propôs que existem dois efeitos do alongamento: agudo e crônico (acumulado). Para ele, agudamente, o alongamento induziria uma diminuição da força muscular, como o que já foi concernido anteriormente. Já sob o aspecto crônico, o autor defende a sua ação contrária, ou seja, benéfica, como uma melhora do desempenho muscular. Isso seria explicado pelo aumento da velocidade e da força de contração, observado em indivíduos que realizaram programas de alongamento associado com fortalecimento durante meses, o que corrobora com os achados deste estudo.

Além de aumentar a AM articular, Pinheiro e Goes (2010), verificaram que o alongamento restaura e/ou mantém o comprimento e o número dos sarcômeros seriados, sendo de fundamental relevância na hiperplasia e hipertrofia das fibras musculares. O ajuste no comprimento do sarcômeros do músculo auxilia-o no desenvolvimento da tensão máxima, tornando, dessa forma, os exercícios de alongamento uma ferramenta terapêutica frequentemente utilizada na reabilitação física e desportiva.

Na variável flexibilidade, avaliada pelo TSA, do GC masculino e feminino, pode-se observar que as medidas da reavaliação, após o período de intervenção, se mantiveram próximas às medidas da avaliação inicial ou encontraram-se abaixo delas. Demonstrando assim que estes indivíduos não ganharam flexibilidade nesse intervalo de tempo, mas sim perderam. Porém, essa perda não apresentou nível de significância estatisticamente para o controle masculino (p=0,68318679) $($ Tabela 3) e feminino ( $\mathrm{p}=0,489208$ ) (Tabela 4). Todavia no GI, masculino e feminino nota-se que após o protocolo de alongamento, os indivíduos desse grupo ganharam flexibilidade, já que os valores da reavaliação ficaram bem acima dos mensurados na avaliação inicial. Este ganho foi significativo estatisticamente para o masculino (p=0,03690391) (Tabela 3) e feminino $(\mathrm{p}=0,024823)$ (Tabela 4).

Aquino et al. (2011), em seus estudos observaram que com o aumento da tolerância ao alongamento, o indivíduo permite a aplicação de uma maior quantidade de força sobre a articulação, possibilitando a obtenção de valores superiores de AM na determinação da flexibilidade. O alongamento produz efeitos imediatos e uma única sessão em indivíduos saudáveis é capaz de melhorar a flexibilidade muscular (amplitude máxima passiva fisiológica) e mobilidade articular.

Salvini et al. (2012), avaliaram o efeito do alongamento na plasticidade do músculo esquelético, os quais mostraram que as fibras musculares se adaptam ao alongamento pela adição de novos sarcômeros em série em suas extremidades, sem alterar a quantidade de tecido conjuntivo. Os resultados científicos observados até o momento indicam que o alongamento é capaz de promover o aumento da síntese proteica. Como o mecanismo de hipertrofia e hiperplasia envolvem aumento da síntese e acúmulo de proteínas, um aumento na expressão de genes musculoespecíficos é necessário (mecanotransdução). 
Tabela 3 - Medidas de flexibilidade e desempenho físico do GC e GI masculino antes e depois* do período de intervenção. Piripiri-PI. ( $\mathrm{n}=20)$.

\begin{tabular}{|c|c|c|c|c|c|}
\hline \multicolumn{6}{|c|}{ Grupo Controle } \\
\hline \multicolumn{2}{|c|}{ FLEXIBILIDADE(cm) } & \multicolumn{4}{|c|}{ DESEMPENHO FÍSICO(cm) } \\
\hline ANTES & DEPOIS & $\mathrm{p}$-valor & ANTES & DEPOIS & $\mathrm{p}$-valor \\
\hline MÉDIA $\pm D P$ & MÉDIA $\pm D P$ & & MÉDIA $\pm \mathrm{DP}$ & MÉDIA $\pm D P$ & \\
\hline $3,41 \pm 10,52$ & $1,59 \pm 9,05$ & $0,68318679 * *$ & $185,1 \pm 24,84$ & $188 \pm 24,36$ & $0,79509957 * *$ \\
\hline \multicolumn{6}{|c|}{ GRUPO INTERVENÇÃO } \\
\hline ANTES & DEPOIS & p-valor & ANTES & DEPOIS & p-valor \\
\hline MÉDIA $\pm D P$ & MÉDIA $\pm D P$ & & 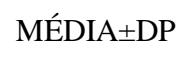 & 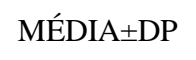 & \\
\hline $4,05 \pm 10,95$ & $14,24 \pm 6,63$ & $0,03690391 * * *$ & $207,20 \pm 20,30$ & $240,80 \pm 26,53$ & $0,00517732 * * *$ \\
\hline
\end{tabular}

*Dados apresentados como média, desvio padrão e p-valor.

**Sem diferença significante antes e depois sem aplicação do alongamento.

***Com diferença significante antes e depois com aplicação do alongamento.

Fonte: Dados da pesquisa (2018).

Tabela 4 - Medidas de flexibilidade e desempenho físico do GC e GI feminino antes e depois* do período de intervenção. Piripiri-PI. (n=20).

\begin{tabular}{|c|c|c|c|c|c|}
\hline \multicolumn{6}{|c|}{ Grupo Controle } \\
\hline \multicolumn{2}{|c|}{ FLEXIBILIDADE(cm) } & \multicolumn{4}{|c|}{ DESEMPENHO FÍSICO $(\mathrm{cm})$} \\
\hline ANTES & DEPOIS & p-valor & ANTES & DEPOIS & p-valor \\
\hline MÉDIA $\pm \mathrm{DP}$ & MÉDIA $\pm D P$ & & MÉDIA $\pm D P$ & MÉDIA $\pm D P$ & \\
\hline$-0,92 \pm 10,35$ & $-3,99 \pm 9,05$ & $0,489208 * *$ & $113,80 \pm 18,21$ & $119,10 \pm 18,73$ & $0,52919 * *$ \\
\hline \multicolumn{6}{|c|}{ GRUPO INTERVENÇÃO } \\
\hline ANTES & DEPOIS & $\mathrm{p}$-valor & ANTES & DEPOIS & $\mathrm{p}$-valor \\
\hline MÉDIA $\pm \mathrm{DP}$ & MÉDIA $\pm D P$ & & MÉDIA $\pm D P$ & MÉDIA $\pm D P$ & \\
\hline$-5,45 \pm 8,49$ & $4,65 \pm 9,07$ & $0,024823 * * *$ & $112,60 \pm 24,71$ & $154,90 \pm 11,53$ & $0,000113895^{* * *}$ \\
\hline
\end{tabular}

*Dados apresentados como média, desvio padrão e p-valor.

**Sem diferença significante antes e depois sem aplicação do alongamento

***Com diferença significante antes e depois com aplicação do alongamento.

Fonte: Dados da pesquisa (2018).

No desempenho físico, avaliado pelo teste do salto horizontal, o GC masculino e feminino apresentaram valores de reavaliação superiores aos encontrados na avaliação inicial, porém como pode-se observar na Tabela 3 (controle masculino) e Tabela 8 (controle feminino), não apresentaram significância estatisticamente ( $\mathrm{p}=0,79509957$; $\mathrm{p}=0,52919$ respectivamente). Este ganho pode ser explicado pelos benefícios adquiridos com a própria prática de AF, mesmo com a abstinência do alongamento, já que todos os indivíduos deste estudo eram ativos há mais de 2 anos. E no GI também houve ganhos, porém, estes foram ainda maiores, se comparados aos do GC, demonstrando que os alongamentos reforçam a AF e aprimoram os benefícios adquiridos com ela. Os dados do GI apresentaram significância estatisticamente para o GI masculino $(\mathrm{p}=0,00517732)$ (Tabela 3) e intervenção feminino $(\mathrm{p}=0,000113895)$ (Tabela 4).

O salto horizontal juntamente com o salto vertical têm sido utilizados com indicadores da potência e força muscular. O salto horizontal, em especial, pode ser utilizado na avaliação e controle do treinamento, com o objetivo de avaliar a potência dos MMII. Segundo os autores, um dos aspectos que é comum ao desempenho do salto é a utilização da energia elástica, 
produzida pelo ciclo de alongamento e encurtamento para produzir força. O alongamento pré-contração é mais eficaz quando todas as fibras da musculatura ou grande parte delas estão treinadas (mais pontes cruzadas formadas), isso é conseguido através do treino associado à técnica de alongamento, a qual proporciona ativação de mais fibras musculares (Coledam, Arruda \& Oliveira, 2013).

A prática de alongamento estático diminui a habilidade de contração muscular isométrica voluntária máxima. As diminuições são amenas e variam de 2 a 5\%. Esta diferença é clinicamente relevante para o atleta de elite, porém irrelevantes para os atletas recreacionais que querem levar uma vida ativa (Shrier, 2011). A diminuição do desempenho muscular após alongamento tem sido documentada pelo consenso crescente de muitos estudos, todavia, essa diminuição é um efeito agudo e temporário da musculatura. A redução do desempenho de $4 \%$ a 30\% foi observada em testes de força máxima e saltos. Queda no desempenho muscular induzida pelo alongamento parece estar igualmente associado à inibição neuromuscular e diminuição da força contrátil e pode ter duração de até uma hora (Knudson, 2013).

Na Tabela 5, quando comparam-se os valores antes do protocolo de alongamento entre os GC e GI, comparando ambos os sexos, percebe-se pela média e o desvio padrão que os valores são próximos e homogêneos, porém quando analisamse os valores após a intervenção, nota-se a discrepância entre as médias pelo desvio padrão, o que indica que os valores mensurados entre os grupos controle e intervenção estão distantes estatisticamente. Dessa forma constata-se a interferência positiva do alongamento nas variáveis avaliadas. No grupo masculino, os valores encontrados apresentam significância estatisticamente para dinamometria de $\operatorname{MSD}(\mathrm{p}=0,00027)$, flexibilidade ( $\mathrm{p}=0,005068)$ e para desempenho físico ( $\mathrm{p}=0,0002)$, mas não para dinamometria de MSE ( $\mathrm{p}=0,06188$ ). Já no grupo feminino, todos os valores encontrados nessa análise são estatisticamente significativos para a dinamometria de MSD e MSE, flexibilidade e desempenho físico ( $\mathrm{p}=0,007004996$; $\mathrm{p}=0,0008 ; \mathrm{p}=0,057829265$ e $\mathrm{p}=0,000120065$, respectivamente).

Di Alencar e Matias (2010), concordam que o encurtamento do sistema muscular, provocado pela prática de AF sem alongamento, gera como consequências a desestabilização da postura, aumento do gasto energético, compressão das fibras nervosas, utilização de fibras musculares compensatórias, aumento das incidências de cãibras e dor, além de prejuízo da técnica nas habilidades esportivas. Exercícios de alongamento são fundamentais para a prevenção e o tratamento de encurtamento muscular. 
Tabela 5 - Medidas de dinamometria, flexibilidade e desempenho físico do GI versus GC masculino e feminino antes e depois* do protocolo de intervenção. Piripiri-PI. $(n=40)$.

\begin{tabular}{|c|c|c|c|c|}
\hline \multicolumn{5}{|c|}{ GC VERSUS GI MASCULINOS } \\
\hline \multirow{2}{*}{ VARIAVEIS } & & \multicolumn{2}{|c|}{ MÉDIA \pm DP } & \multirow{2}{*}{$\mathrm{p}$-valor } \\
\hline & & ANTES & DEPOIS & \\
\hline \multirow[t]{2}{*}{ DINAMOMETRIA } & MSD & $5033,78 \pm 174,46$ & $5186,43 \pm 391,16$ & $0,00027 * *$ \\
\hline & MSE & $4963,40 \pm 7,88$ & $5094,50 \pm 382,85$ & $0,06188 * * *$ \\
\hline FLEXIBILIDADE & & $3,73 \pm 0,45$ & $7,92 \pm 8,94$ & $0,005068^{* *}$ \\
\hline DESEMPENHO FÍSICO & & $196,15 \pm 15,63$ & $214,40 \pm 37,34$ & $0,0002 * *$ \\
\hline \multicolumn{5}{|c|}{ GC VERSUS GI FEMININOS } \\
\hline \multirow{2}{*}{ VARIAVEIS } & & MÉDIA \pm DP & & p-valor \\
\hline & & ANTES & DEPOIS & \\
\hline \multirow[t]{2}{*}{ DINAMOMETRIA } & MSD & $4348,80 \pm 212,47$ & $4702,92 \pm 371,70$ & $0,007004996 * *$ \\
\hline & MSE & $4562,24 \pm 52,28$ & $4830,56 \pm 434,28$ & $0,00008 * *$ \\
\hline FLEXIBILIDADE & & $-3,18 \pm 3,21$ & $0,33 \pm 6,11$ & $0,057829265^{* *}$ \\
\hline DESEMPENHO FÍSICO & & $113,20 \pm 0,85$ & $137,00 \pm 25,31$ & $0,000120065^{* *}$ \\
\hline
\end{tabular}

*Dados apresentados como média, desvio padrão e p-valor.

**Com diferença significante antes e depois com aplicação do alongamento.

***Sem diferença significante antes e depois com aplicação do alongamento.

Fonte: Dados da pesquisa (2018).

Diversos estudos de Salvador et al. (2012), têm investigado o desempenho motor de homens e mulheres em contrações isométricas, isocinéticas e dinâmicas, bem como a capacidade de resistência à fadiga. Em um de seus trabalhos, analisou o comportamento de homens e mulheres em séries múltiplas de exercícios executados com pesos livres para os diferentes segmentos corporais. Diferenças na força muscular entre os sexos, tanto em termos absolutos quanto relativos, também foram identificadas nesta investigação, em todos os exercícios analisados, com os homens apresentando valores superiores aos das mulheres. $\mathrm{O}$ único aspecto em que as mulheres superaram os homens foi na resistência à fadiga, porém isso diminui à medida que a intensidade do esforço aumenta.

Fazer alongamentos associados à AF, principalmente antes à sua prática, faz com que surjam fibras musculares novas e, portanto, não educadas, provocando descoordenação e aumentando consideravelmente o risco de lesão. O que não acontece se estas fibras musculares forem ativadas periodicamente através do alongamento. Dessa maneira, o ventre muscular estará sendo estimulado de forma integral, garantindo uma performance muscular completa e eficaz (Gergley, 2013).

Constatou-se então que o alongamento acumulado (efeitos crônicos) auxilia no desempenho motor de indivíduos ativos, tanto no aspecto celular recrutando mais sarcômeros, quanto no aspecto tecidual ativando mais fibras musculares, exigindo a musculatura de forma integral, aprimorando e ampliando assim, os resultados adquiridos com a AF.

\section{Conclusão}

O desenvolvimentro do presente estudo possibilitou uma análise do impacto do alongamento na flexibilidade e força muscular, demonstrando ser um grande aliado dos indivíduos praticantes de AF. A técnica pode potencializar o treinamento, sob o aspecto preventivo e preparatório do exercício, permitindo que seu praticante tenha um maior aproveitamento e obtenha maiores resultados. 
Ao realizar as intervenções no mesmo lugar onde os participantes estavam e em um período de um mês, verificou-se que as partes mais complexas e desgastantes do processo, que são deslocamento dos participantes, estresse à intervenção foram evitados e apuração dos resultados foi realizada com êxito. Permitindo assim que os objetivos propostos fossem realmente alcançados.

Destaca-se que o estudo apresentou algumas limitações como literatura escassa de trabalhos que avaliassem o impacto do alongamento em diferentes variáveis quantitativas. Dada a importância do assunto, torna-se necessário o desenvolvimento de novos estudos, afim de obter mais dados e reforçar, bem como validar e promover o uso do alongamento associado a prática de AF.

\section{Referências}

Aquino, C. et al. (2011). Análise da confiabilidade de um método de mensuração do ângulo de pico de torque ativo dos isquiossurais. Revista Brasileira de Fisioterapia, 11 (2).

Camargo, M. R. de. et al. (2009). Avaliação da Força Muscular Isométrica do tornozelo. Dinamometria: descrição de uma nova técnica. Revista Brasileira de Ciências da Saúde. 13 (2).

Coledam, D. H. C., Arruda, G. A. \& Oliveira, A. R. (2013). Efeitos de um programa de exercícios no desempenho de crianças nos testes de flexibilidade e impulsão vertical. Motriz: Revista de Educação. Física. 18 (3).

Di Alencar, T. A. M. \& Matias, K. F. S. (2010). Princípios fisiológicos do aquecimento e alongamento muscular na atividade esportiva. Revista Brasileira de. Medicina do Esporte. Niterói, 16 (3).

Falk, P. R. A. \& Pereira, D. P. (2009). Utilização do salto horizontal como comparativo no método de avaliação de força de membros inferiores de alunos da escola de futebol doGrêmio de Lages, SC. Lecturas, Educación Física y Deportes, Revista Digital. Buenos Aires. 14 (139).

Gergley, J. C. (2013). Acute Effect Of Passive Static Stretching On Lower-Body Strength In Moderately Trained Men. The Journal of Strength and Conditioning Research. 27 (4).

Kisner, C. \& Colby, L. A. (2009). Exercícios Terapêuticos. Manole.

Knudson, D. V. (2013). Warm-up and Flexibility. In: Chandler, T. J., \& Brown, L. E. Conditioning for Strength and Human Performance. Philadelphia, PA: Lippincott-Williams \& Wilkins, Journal of the Canadian Chiropractic Association. 57(1): 94.

Lustosa, L. P. et al. (2010). Impacto do alongamento estático no ganho de força muscular dos extensores de joelho em idosas da comunidade após um programa de treinamento. Revista Brasileira de Fisioterapia. 14 (6).

Maciel, M. G. (2010). Atividade física e funcionalidade do idoso. Motriz: Revista de Educação Física. 16 (4).

Morcelli, M. H., Oliveira, J. M. C. A. \& Navega, M. T. (2013). Comparação do alongamento estático, balístico e contrair-relaxar nos músculos isquiotibiais. Fisioterapia e Pesquisa. 20 (3).

Oliveira, A. et al. (2013). Relação dos saltos vertical, horizontal e sêxtuplo com a agilidade e velocidade em crianças. Revista Bras. Med. Esporte. 27 (1).

Paulo, A. C. et al. (2012). Efeito agudo dos exercícios de flexibilidade no desempenho de força máxima e resistência de força de membros inferiores e superiores Motriz: Revista de Educação Fisica. 18 (2).

Pinheiro, I. M. \& Goes, A. L. B. (2010). Efeitos imediatos do alongamento em diferentes posicionamentos. Fisioterapia em Movimento. 23 (4).

Ribeiro, C. C. A. et al. (2010). Nível de flexibilidade obtida pelo teste de sentar e alcançar a partir de estudo realizado na grande São Paulo. Revista Brasileira de Cineantropometria e Desempenho Humano. 12 (6).

Salvador, E. P. et al. (2012). Comparação entre o desempenho motor de homens e mulheres em séries múltiplas de exercícios com pesos. Revista Brasileira de Medicina do Esporte, 11 (5).

Salvini, T. F. et al. (2012). Efeitos da eletroestimulação e do alongamento muscular sobre a adaptação do músculo desnervado: implicações para a fisioterapia. Revista Brasileira de Fisioterapia. 16 (3).

Shrier, I. (2014). Does stretching improve performance? A systematic and critical review of the literature. Clinical Journal of Sport Medicine. 14 (5).

Shrier, I. (2011). When and Whom to Stretch? Phys Sportsmed. 33(3):22-6.

Soares, A. V. et al. (2011). Dinamometria de preensão manual como parâmetro de avaliação funcional do membro superior de pacientes hemiparéticos por acidente vascular cerebral. Fisioter. e Pesqui. 18 (4). 